q-alg/9706014

\title{
A Jordanian quantum two-photon/Schrödinger algebra
}

\author{
Angel Ballesteros ${ }^{\dagger}$, Francisco J. Herranz ${ }^{\dagger}$ and Preeti Parashar ${ }^{\ddagger}$ \\ † Departamento de Fúsica, Universidad de Burgos \\ Pza. Misael Bañuelos, E-09001-Burgos, Spain \\ $¥$ Department of Mathematics, Texas A \& $M$ University \\ College Station, Texas 7r843, USA
}

\begin{abstract}
A non-standard quantum deformation of the two-photon algebra $h_{6}$ is constructed, and its quantum universal $R$-matrix is given. Representations of this new quantum algebra are studied on the Fock space and translated into Fock-Bargmann realizations that provide a direct formalism for the definition of deformed states of light. Finally, the isomorphism between $h_{6}$ and the $(1+1)$ Schrödinger algebra is used to introduce a new (non-standard) Hopf algebra deformation of this latter symmetry algebra.
\end{abstract}




\section{Introduction}

The single-mode radiation field Hamiltonian that describes the generation of a squeezed coherent state is given by [1]:

$$
H=\hbar \omega\left(a_{+} a_{-}+\frac{1}{2}\right)+f_{1}(t) a_{+}+f_{1}(t)^{*} a_{-}+f_{2}(t) a_{+}^{2}+f_{2}(t)^{*} a_{-}^{2},
$$

where $a_{-}$and $a_{+}$are the generators of the boson algebra: $\left[a_{-}, a_{+}\right]=1$. The dynamical symmetry algebra of this Hamiltonian involving two-photon processes is the "two-photon algebra" $h_{6}$, a six dimensional non-semisimple Lie algebra generated by

$$
\begin{array}{lll}
N=a_{+} a_{-} & A_{+}=a_{+} & A_{-}=a_{-} \\
M=1 & B_{+}=a_{+}^{2} & B_{-}=a_{-}^{2}
\end{array}
$$

where $N$ is the number operator and $M$ is a central generator. The commutation rules among these generators are

$$
\begin{array}{lll}
{\left[N, A_{+}\right]=A_{+}} & {\left[N, A_{-}\right]=-A_{-}} & {\left[A_{-}, A_{+}\right]=M} \\
{\left[N, B_{+}\right]=2 B_{+}} & {\left[N, B_{-}\right]=-2 B_{-}} & {\left[B_{-}, B_{+}\right]=4 N+2 M} \\
{\left[A_{+}, B_{-}\right]=-2 A_{-}} & {\left[A_{+}, B_{+}\right]=0} & {[M, \cdot]=0} \\
{\left[A_{-}, B_{+}\right]=2 A_{+}} & {\left[A_{-}, B_{-}\right]=0 .} &
\end{array}
$$

Three relevant subalgebras of $h_{4}$ arise: the Heisenberg-Weyl algebra $h_{3}$ (generated by $\left.A_{+}, A_{-}, M\right)$, the oscillator algebra $h_{4}$ (which is $h_{3}$ enlarged with the number generator $N$ ) and the $s u(1,1)$ algebra (defined by $B_{+}^{\prime}, B_{-}^{\prime}, N^{\prime}$, where $N^{\prime}=(N+$ $\left.M / 2) / 2, B_{ \pm}^{\prime}=B_{ \pm} / 2\right)$. Thus we have the sequence $h_{3} \subset h_{4} \subset h_{6}$. The essential role played by these three symmetries in the algebraic description of coherent, squeezed and intelligent states of light is well-known, and it has been recently unified within a two-photon algebra approach in [2]. Our paper introduces a quantum deformation of the two photon algebra and presents its potential algebraic abilities in order to construct deformed (and non-classical) states of light.

In section 2, a non-standard quantum (Hopf algebra) deformation of $h_{6}$ is obtained by making use of the Jordanian deformation of its subalgebra $h_{4}$ [3]. In this example, it will become clear that although $B_{+}$coincides with the square of $A_{+}$in the representation (1.2), this relation is no longer true in general. In spite of this fact, it is also shown that the quantum universal $R$-matrix associated to the original $h_{4}$ deformation is just the quantum $R$-matrix for the two-photon quantization.

In general, applications in Quantum Optics of all the algebras mentioned before, came initially from representations like (1.2) in terms of creation and annihilation operators (boson realizations). Section 3 deals with the corresponding deformation of the boson realization (1.2). From an algebraic point of view, the resultant representation can be translated into a realization in terms of operators acting on the Hilbert space of entire analytic functions where the construction of [2] holds. In this context, it is shown how the linear differential operators of the undeformed case can be transformed after quantization either into more complicated differential 
operators or into differential-difference ones. The different role played by $A_{+}$and $A_{-}$after quantization is emphasized, and the physical inequivalence of algebraically equivalent quantizations is stressed.

The second main purpose of the work is to show the usefulness (that, to our knowledge, has not been used yet) of the isomorphism between the two-photon algebra and the $(1+1)$ Schrödinger algebra. In particular, we shall present in the last section a new quantum deformation of the latter algebra endowed with a Hopf algebra structure, something that is left out in the previous $q$-deformations so far obtained [4, 5]. As a byproduct, this isomorphism shows that the extended $(1+1)$ Galilei algebra is also a relevant subalgebra of $h_{6}$.

\section{The deformation}

The non-standard (or Jordanian) quantum deformation of the oscillator algebra $h_{4}$ was introduced in [3] (the essentials of Lie bialgebra quantizations needed in order to derive the following results can be found there; a comprehensive presentation of the subject is given in [6]). The starting point of the construction was the (coboundary) Lie bialgebra $\left(h_{4}, \delta(r)\right)$ defined by the classical $r$-matrix

$$
r=z N \wedge A_{+}
$$

(which is a solution of the classical Yang-Baxter equation) where $N$ and $A_{+}$belong to $h_{4}$. Therefore, the embedding $h_{4} \subset h_{6}$ allows us to consider (2.1) as the generating object for a Jordanian deformation of $h_{6}$ by taking $N$ and $A_{+}$as generators for this algebra. The cocommutators of the two-photon Lie bialgebra $\left(h_{6}, \delta(r)\right)$ are obtained from the relation $\delta(X)=[1 \otimes X+X \otimes 1, r]$ and read

$$
\begin{aligned}
& \delta\left(A_{+}\right)=0 \quad \delta(M)=0 \\
& \delta(N)=z N \wedge A_{+} \quad \delta\left(B_{+}\right)=-2 z B_{+} \wedge A_{+} \\
& \delta\left(A_{-}\right)=z\left(A_{-} \wedge A_{+}+N \wedge M\right) \\
& \delta\left(B_{-}\right)=2 z\left(B_{-} \wedge A_{+}+N \wedge A_{-}\right) .
\end{aligned}
$$

Note that $\delta\left(B_{-}\right)$contains the term $N \wedge A_{-}$, which involves two "non-primitive" generators. As a consequence (see [3]) it is not possible to "exponentiate" directly the first order in the deformation given by the Lie bialgebra (2.2) in order to obtain the full coproduct of the quantum deformation we are looking for. However, by assuming that the right quantum structure is not far away from the exponentiation of (2.2), the following coassociative coproduct for the quantum two-photon algebra $U_{z}\left(h_{6}\right)$ can be obtained:

$$
\begin{aligned}
& \Delta\left(A_{+}\right)=1 \otimes A_{+}+A_{+} \otimes 1 \quad \Delta(M)=1 \otimes M+M \otimes 1 \\
& \Delta(N)=1 \otimes N+N \otimes e^{z A_{+}} \quad \Delta\left(B_{+}\right)=1 \otimes B_{+}+B_{+} \otimes e^{-2 z A_{+}} \\
& \Delta\left(A_{-}\right)=1 \otimes A_{-}+A_{-} \otimes e^{z A_{+}}+z N \otimes e^{z A_{+}} M \\
& \Delta\left(B_{-}\right)=1 \otimes B_{-}+B_{-} \otimes e^{2 z A_{+}}+z N \otimes e^{z A_{+}}\left(A_{-}-z M N\right)-z A_{-} \otimes e^{z A_{+}} N .
\end{aligned}
$$


Deformed commutation rules consistent with (2.3) are given in the form:

$$
\begin{aligned}
& {\left[N, A_{+}\right]=\frac{e^{z A_{+}-1}}{z} \quad\left[N, A_{-}\right]=-A_{-} \quad\left[A_{-}, A_{+}\right]=M e^{z A_{+}}} \\
& {\left[N, B_{+}\right]=2 B_{+} \quad\left[N, B_{-}\right]=-2 B_{-}-z A_{-} N \quad[M, \cdot]=0} \\
& {\left[B_{-}, B_{+}\right]=2\left(1+e^{-z A_{+}}\right) N+2 M-2 z A_{-} B_{+}} \\
& {\left[A_{+}, B_{-}\right]=-\left(1+e^{z A_{+}}\right) A_{-}+z e^{z A_{+}} M N \quad\left[A_{+}, B_{+}\right]=0} \\
& {\left[A_{-}, B_{+}\right]=2 \frac{1-e^{-z A_{+}}}{z} \quad\left[A_{-}, B_{-}\right]=-z A_{-}^{2} .}
\end{aligned}
$$

Finally, counit $\epsilon$ and antipode $\gamma$ for $U_{z}\left(h_{6}\right)$ read

$$
\begin{aligned}
& \epsilon(X)=0 \quad \text { for } X \in\left\{N, A_{+}, A_{-}, B_{+}, B_{-}, M\right\} \\
& \gamma\left(A_{+}\right)=-A_{+} \quad \gamma(M)=-M \\
& \gamma(N)=-N e^{-z A_{+}} \quad \gamma\left(B_{+}\right)=-B_{+} e^{2 z A_{+}} \\
& \gamma\left(A_{-}\right)=-A_{-} e^{-z A_{+}}+z N M e^{-z A_{+}} \\
& \gamma\left(B_{-}\right)=-B_{-} e^{-2 z A_{+}}-z A_{-} e^{-2 z A_{+}} .
\end{aligned}
$$

Note that the non-standard quantum oscillator algebra $U_{z}\left(h_{4}\right)$ is a Hopf subalgebra of $U_{z}\left(h_{6}\right)$. The quantum universal $R$-matrix for $U_{z}\left(h_{4}\right)$

$$
\mathcal{R}=\exp \left\{-z A_{+} \otimes N\right\} \exp \left\{z N \otimes A_{+}\right\}
$$

was found in [3]. In fact, this element $\mathcal{R}$ can be proven to be the quantum $R$-matrix for $U_{z}\left(h_{6}\right)$ when $A_{+}$and $N$ are considered as generators of the latter algebra (see Appendix A). Recall that its classical counterpart (2.1) has been used to define the whole Lie bialgebra (2.2), and that this kind of embedding has been shown to preserve the quantum $R$-matrix for the initial subalgebra in other examples of non-standard deformations (see [7, 8]).

\section{Representation theory}

The physical relevance of the two-photon algebra comes from the Hamiltonians of the type (1.1), in which a certain boson representation for $h_{6}$ gives a dynamical

symmetry of the problem. Let us generalize such kind of representations to the quantum case.

\subsection{Quantum boson realizations}

A realization of $h_{6}(1.3)$ in terms of the boson generators $a_{-}, a_{+}$is given by (1.2). When the previous boson operators act on the number states Hilbert space spanned by $\{|m\rangle\}_{m=0}^{\infty}$ the action of the two-photon operators on these states is

$$
\begin{aligned}
& N|m\rangle=m|m\rangle \quad M|m\rangle=|m\rangle \\
& A_{+}|m\rangle=\sqrt{m+1}|m+1\rangle \quad A_{-}|m\rangle=\sqrt{m}|m-1\rangle \\
& B_{+}|m\rangle=\sqrt{(m+1)(m+2)}|m+2\rangle \quad B_{-}|m\rangle=\sqrt{m(m-1)}|m-2\rangle .
\end{aligned}
$$


A part of this representation can be deformed by considering the results given in [9] concerning the subalgebra defined by the quantum oscillator $U_{z}\left(h_{4}\right)$. In particular, by setting $\beta=0, \delta=1$, and by replacing $2 z \rightarrow z$ in those results, we get the realization:

$$
N=\frac{e^{z a_{+}}-1}{z} a_{-} \quad A_{+}=a_{+} \quad A_{-}=e^{z a_{+}} a_{-} \quad M=1 .
$$

The remaining generators are easily found to be realized as

$$
B_{+}=\left(\frac{1-e^{-z a_{+}}}{z}\right)^{2} \quad B_{-}=e^{z a_{+}} a_{-}^{2} .
$$

Therefore, the classical identification $B_{+}=A_{+}^{2}$ is no longer valid in the quantum case. Moreover, the coproduct (2.3) for $\Delta\left(B_{+}\right)$is strongly different from $\Delta\left(A_{+}\right)^{2}$ (in fact, this property appears at a non-deformed level aswell [10]).

Now, by taking into account that

$$
e^{z a_{+}}|m\rangle=|m\rangle+\sum_{k=1}^{\infty} \frac{z^{k}}{k !} \sqrt{\frac{(m+k) !}{m !}}|m+k\rangle
$$

the action of the generators of $U_{z}\left(h_{6}\right)$ on the number states $\{|m\rangle\}_{m=0}^{\infty}$ reads

$$
\begin{aligned}
A_{+}|m\rangle= & \sqrt{m+1}|m+1\rangle \quad M|m\rangle=|m\rangle \\
A_{-}|m\rangle= & \sqrt{m}|m-1\rangle+m \sum_{k=0}^{\infty} \frac{z^{k+1}}{(k+1) !} \sqrt{\frac{(m+k) !}{m !}}|m+k\rangle \\
N|m\rangle= & m|m\rangle+m \sum_{k=1}^{\infty} \frac{z^{k}}{(k+1) !} \sqrt{\frac{(m+k) !}{m !}}|m+k\rangle \\
B_{+}|m\rangle= & \sqrt{(m+1)(m+2)}|m+2\rangle \\
& +\sum_{k=1}^{\infty}\left(-2+2^{k+2}\right) \frac{(-z)^{k}}{(k+2) !} \sqrt{\frac{(m+k+2) !}{m !}}|m+k+2\rangle \\
B_{-}|m\rangle= & \sqrt{m(m-1)}|m-2\rangle+z \sqrt{m(m-1)}|m-1\rangle \\
& +m(m-1) \sum_{k=0}^{\infty} \frac{z^{k+2}}{(k+2) !} \sqrt{\frac{(m+k) !}{m !}}|m+k\rangle .
\end{aligned}
$$

The numbers $\left\langle m|X| m^{\prime}\right\rangle$ where $\left\langle m \mid m^{\prime}\right\rangle=\delta_{m, m^{\prime}}$ give rise to the matrix elements of this infinite-dimensional representation; explicitly,

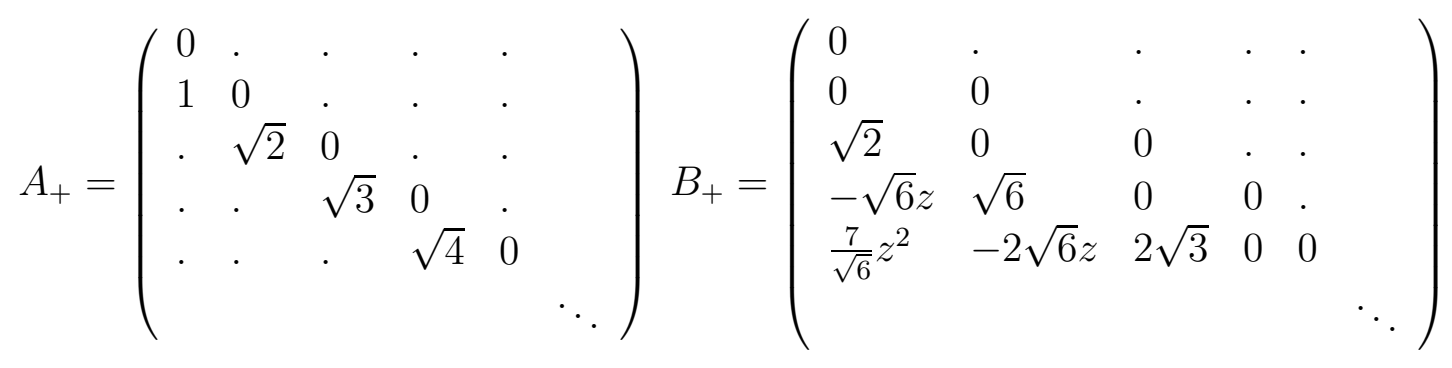




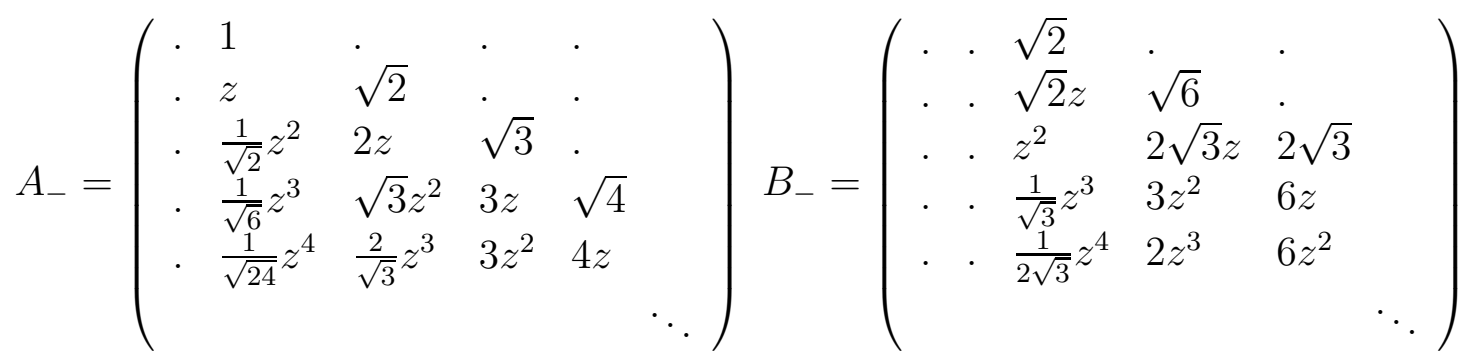

$$
\begin{aligned}
& N=\left(\begin{array}{llllll}
\cdot & \cdot & \cdot & \cdot & \cdot & \\
\cdot & 1 & \cdot & \cdot & \cdot & \\
\cdot & \frac{1}{\sqrt{2}} z & 2 & \cdot & \cdot & \\
\cdot \frac{1}{\sqrt{6}} z^{2} & \sqrt{3} z & 3 & \cdot & \\
\cdot & \frac{1}{\sqrt{24}} z^{3} & \frac{2}{\sqrt{3}} z^{2} & 3 z & 4 & \\
& & & & & \cdot
\end{array}\right) \quad M=\left(\begin{array}{cccccc}
1 & \cdot & \cdot & \cdot & \cdot & \\
\cdot & 1 & \cdot & \cdot & \cdot & \\
\cdot & \cdot & 1 & \cdot & \cdot & \\
\cdot & \cdot & \cdot & 1 & \cdot & \\
\cdot & \cdot & \cdot & \cdot & 1 & \\
& & & & & \ddots
\end{array}\right)
\end{aligned}
$$

Note the characteristic appearance of monomials in $z$ as entries of the deformed matrices. In this respect, see [9, 11, 12, 13, 14, 15] for representations of nonstandard quantum algebras where this feature appears recurrently.

\subsection{Fock-Bargmann realizations}

The deformed boson realization $(3.2-3.3)$ can be immediately translated into differential operators acting on the the space of entire analytic functions $f(\alpha)$ (the Fock-Bargmann (FB) representation [16]):

$$
\begin{aligned}
& N=\frac{e^{z \alpha}-1}{z} \frac{d}{d \alpha} \quad A_{+}=\alpha \quad A_{-}=e^{z \alpha} \frac{d}{d \alpha} \quad M=1 \\
& B_{+}=\left(\frac{1-e^{-z \alpha}}{z}\right)^{2} \\
& B_{-}=e^{z \alpha} \frac{d^{2}}{d \alpha^{2}} .
\end{aligned}
$$

These expressions would be the starting point for a general study of deformed states of light generated by the quantum deformation introduced here (see [2] for the classical construction). However, there also exists another set of operators defined on the FB space and algebraically linked to this deformation. It is easy to check that the following automorphism of $h_{6}$

$$
\begin{array}{lll}
N \rightarrow-N & A_{+} \rightarrow-A_{-} & A_{-} \rightarrow-A_{+} \\
M \rightarrow-M & B_{+} \rightarrow-B_{-} & B_{-} \rightarrow-B_{+}
\end{array}
$$

and the transformation of the deformation parameter

$$
z \rightarrow-z
$$

transforms the Lie bialgebra (2.2) into

$$
\begin{aligned}
& \delta\left(A_{-}\right)=0 \quad \delta(M)=0 \\
& \delta(N)=z N \wedge A_{-} \quad \delta\left(B_{-}\right)=-2 z B_{-} \wedge A_{-} \\
& \delta\left(A_{+}\right)=z\left(A_{+} \wedge A_{-}+N \wedge M\right) \\
& \delta\left(B_{+}\right)=2 z\left(B_{+} \wedge A_{-}+N \wedge A_{+}\right) .
\end{aligned}
$$


Therefore, (3.10) and (2.2) are equivalent as Lie bialgebras, and the quantization of the former leads to expressions that can be deduced from the ones given in section 2 by making use of the automorphism (3.8) and (3.9). The essential feature of this deformation is that now $A_{-}$and $M$ are the primitive generators. We shall write only the set of resultant deformed commutation rules:

$$
\begin{aligned}
& {\left[N, A_{-}\right]=-\frac{e^{z A_{-}-1}}{z} \quad\left[N, A_{+}\right]=A_{+} \quad\left[A_{-}, A_{+}\right]=M e^{z A_{-}}} \\
& {\left[N, B_{-}\right]=-2 B_{-} \quad\left[N, B_{+}\right]=2 B_{+}+z A_{+} N \quad[M, \cdot]=0} \\
& {\left[B_{+}, B_{-}\right]=-2\left(1+e^{-z A_{-}}\right) N-2 M+2 z A_{+} B_{-}} \\
& {\left[A_{-}, B_{+}\right]=\left(1+e^{z A_{-}}\right) A_{+}-z e^{z A_{-}} M N \quad\left[A_{-}, B_{-}\right]=0} \\
& {\left[A_{+}, B_{-}\right]=-2 \frac{1-e^{-z A_{-}}}{z} \quad\left[A_{+}, B_{+}\right]=z A_{+}^{2} .}
\end{aligned}
$$

The corresponding FB realization for (3.11) reads (we write $\frac{d}{d \alpha}=d_{\alpha}$ ):

$$
\begin{array}{ll}
N=\alpha\left(\frac{e^{z d_{\alpha}}-1}{z}\right) & A_{+}=\alpha e^{z d_{\alpha}} \quad A_{-}=d_{\alpha} \quad M=1 \\
B_{+}=\left(\alpha^{2}+z \alpha\right) e^{z d_{\alpha}} & B_{-}=\left(\frac{1-e^{-z d_{\alpha}}}{z}\right)^{2} .
\end{array}
$$

The deformed boson realization would be obtained by substituting $d_{\alpha}=a_{-}$and $\alpha=a_{+}$. Note that (3.12) is a differential-difference realization; in particular, the action

$$
\left(\frac{e^{z d_{\alpha}}-1}{z}\right) f(\alpha)=\frac{f(\alpha+z)-f(\alpha)}{z},
$$

corresponds to a discrete derivative. Therefore, (3.12) can be thought of as a certain discretization of the usual FB representation.

From a physical point of view, relevant states of the radiation field appear as eigenfunctions of the generators of the two-photon algebra in the FB representation [2]. Therefore, the two (algebraically equivalent) quantum deformations of $h_{6}$ presented here give rise to different deformed eigenproblems on the space of analytic functions. This procedure seems to be the most natural way for generating Jordanian (and, in general, quantum algebra) analogues of coherent, squeezed and intelligent states. A detailed analysis of this construction and of its physical contents will be presented elsewhere, but we can advance here that the former (3.7) ( $A_{+}$primitive) would originate a class of smooth deformed states, and the latter (3.12) ( $A_{-}$primitive) will be linked to a set of states including some intrinsic discretization. In both cases, the precise shape of the deformation is a consequence of the compatibility with the deformed composition rule of representations given by the corresponding quantum coproduct. 


\section{A quantum (1+1) Schrödinger algebra}

The $(1+1)$ dimensional Schrödinger algebra $\mathcal{S}(1+1)$ 17, 18 is a six-dimensional Lie algebra generated by $H$ (time translation), $P$ (space translation), $K$ (Galilean boost), $D$ (dilation), $C$ (conformal transformation) and $M$ (mass); it is endowed with the following commutation rules:

$$
\begin{array}{lll}
{[D, P]=-P} & {[D, K]=K} & {[K, P]=M} \\
{[D, H]=-2 H} & {[D, C]=2 C} & {[H, C]=D} \\
{[K, H]=P} & {[K, C]=0} & {[M, \cdot]=0} \\
{[P, C]=-K} & {[P, H]=0 .} &
\end{array}
$$

The subalgebra generated by $\{H, P, K, M\}$ defines a $(1+1)$ extended Galilei algebra and the one spanned by $\{D, C, H\}$ closes as an $s l(2, \mathbb{R})$ structure.

The physical interest in $\mathcal{S}(1+1)$ comes from the fact that 4.1 is the Lie algebra of the symmetry group of the $(1+1)$ dimensional Schrödinger equation. Recently, the symmetry analysis of some differential-difference generalizations of this outstanding equation has originated various $q$-analogues of $\mathcal{S}(1+1)$, none of them endowed with a known Hopf algebra structure [4, 5]. However, an isomorphism between $\mathcal{S}(1+1)$ and $h_{6}$ [19], can be explicitly given as follows:

$$
D=-N-\frac{1}{2} M \quad P=A_{+} \quad K=A_{-} \quad H=\frac{1}{2} B_{+} \quad C=\frac{1}{2} B_{-}
$$

keeping $M$ as the central generator for both algebras. Therefore, starting from the results presented in previous sections and by using (4.2), it is immediate to obtain a complete (non-standard) quantum deformation of $\mathcal{S}(1+1)$. Its Lie bialgebra is provided by the classical $r$-matrix

$$
r=z P \wedge D+\frac{z}{2} P \wedge M
$$

with cocommutators given by

$$
\begin{aligned}
& \delta(P)=0 \quad \delta(M)=0 \quad \delta(H)=-2 z H \wedge P \\
& \delta(K)=z(K \wedge P-D \wedge M) \quad \delta(D)=z\left(D \wedge P+\frac{1}{2} M \wedge P\right) \\
& \delta(C)=z\left(2 C \wedge P+K \wedge D+\frac{1}{2} K \wedge M\right) .
\end{aligned}
$$

The coproduct and the commutation rules of the Hopf algebra $U_{z}(\mathcal{S}(1+1))$ are

$$
\begin{aligned}
& \Delta(P)=1 \otimes P+P \otimes 1 \quad \Delta(M)=1 \otimes M+M \otimes 1 \\
& \Delta(H)=1 \otimes H+H \otimes e^{-2 z P} \\
& \Delta(K)=1 \otimes K+K \otimes e^{z P}-z\left(D+\frac{1}{2} M\right) \otimes e^{z P} M \\
& \Delta(D)=1 \otimes D+D \otimes e^{z P}+\frac{1}{2} M \otimes\left(e^{z P}-1\right) \\
& \Delta(C)=1 \otimes C+C \otimes e^{2 z P}-\frac{z}{2}\left(D+\frac{1}{2} M\right) \otimes e^{z P}\left(K+z\left(D+\frac{1}{2} M\right) M\right)
\end{aligned}
$$




$$
\begin{aligned}
+\frac{z}{2} K & \otimes e^{z P}\left(D+\frac{1}{2} M\right), \\
{[D, P] } & =\frac{1-e^{z P}}{z} \quad[D, K]=K \quad[K, P]=M e^{z P} \\
{[D, H] } & =-2 H \quad[D, C]=2 C-\frac{z}{2} K\left(D+\frac{1}{2} M\right) \\
{[H, C] } & =\frac{1}{2}\left(1+e^{-z P}\right)\left(D+\frac{1}{2} M\right)-\frac{1}{2} M+z K H \\
{[K, H] } & =\frac{1-e^{-z P}}{z} \quad[K, C]=-\frac{z}{2} K^{2} \quad[M, \cdot]=0 \\
{[P, C] } & =-\frac{1}{2}\left(1+e^{z P}\right) K-\frac{z}{2} e^{z P} M\left(D+\frac{1}{2} M\right) \quad[P, H]=0 .
\end{aligned}
$$

Note that neither $\{H, P, K, M\}$ (the extended Galilei algebra) nor $\{D, C, H\}$ define a Hopf subalgebra of this deformation. However, the generators $\{D, P, K, M\}$ do close as a Hopf subalgebra (they were the oscillator generators in $h_{6}$ ). Finally, the (factorized) universal $R$-matrix for $U_{z}(\mathcal{S}(1+1))$ is written as

$$
\mathcal{R}=\exp \{z P \otimes D\} \exp \left\{\frac{z}{2} P \otimes M\right\} \exp \left\{-\frac{z}{2} M \otimes P\right\} \exp \{-z D \otimes P\} .
$$

This connection between the two-photon and the $(1+1)$ Schrödinger algebras deserves further analysis. From it, the extended $(1+1)$ Galilei algebra appears as another relevant subalgebra of $h_{6}$ and, in turn, the oscillator algebra $h_{4}$ can be embedded within $\mathcal{S}(1+1)$. On the other hand, the isomorphism (4.2) exhibits again, in the geometrical language of the Schrödinger symmetry, the physical inequivalence between the (algebraically equivalent) quantizations (2.4) and (3.11) shown in section 3 by constructing FB realizations: the first deformation makes $A_{+}=P$ the primitive generator, a role played in the second one by the boost $A_{-}=K$.

\section{Acknowledgments}

A.B. and F.J.H. have been partially supported by DGICYT (Project PB94-1115) from the Ministerio de Educación y Cultura de España and by Junta de Castilla y León (Projects CO1/396 and CO2/297). P.P. wishes to thank Prof. Edward Letzter for hospitality at Texas A \& M University.

\section{Appendix A}

As it was shown in [3] the element (2.7) fulfills both the quantum Yang-Baxter equation and the property

$$
\mathcal{R} \Delta(X) \mathcal{R}^{-1}=\sigma \circ \Delta(X) \quad \text { for } X \in\left\{N, A_{+}, A_{-}, M\right\}
$$


where $\sigma$ is the flip operator: $\sigma(a \otimes b)=b \otimes a$. Since $U_{z}\left(h_{4}\right) \subset U_{z}\left(h_{6}\right)$ we only need to prove that $\mathcal{R}$ also satisfies (A.1) for the two remaining generators $B_{+}$and $B_{-}$. We shall make use of the formula

$$
e^{f} \Delta(X) e^{-f}=\Delta(X)+\sum_{n=1}^{\infty} \frac{1}{n !}\left[f,[\ldots[f, \Delta(X)]]^{n)} \ldots\right]
$$

where the superindex $n$ ) means $n$ commutators. To begin with we take $X \equiv B_{+}$ and $f \equiv z N \otimes A_{+}$. Straightforward computations lead to

$$
\left[z N \otimes A_{+},\left[\ldots\left[z N \otimes A_{+}, \Delta\left(B_{+}\right)\right]\right]^{n)} \ldots\right]=B_{+} \otimes e^{-2 z A_{+}}\left(2 z A_{+}\right)^{n} \quad n \geq 1
$$

so that

$$
\begin{aligned}
e^{z N \otimes} & A_{+} \Delta\left(B_{+}\right) e^{-z N \otimes A_{+}}=\Delta\left(B_{+}\right)+\sum_{n=1}^{\infty} B_{+} \otimes e^{-2 z A_{+}} \frac{\left(2 z A_{+}\right)^{n}}{n !} \\
& =1 \otimes B_{+}+B_{+} \otimes e^{-2 z A_{+}}+B_{+} \otimes e^{-2 z A_{+}}\left(e^{2 z A_{+}}-1\right) \\
& =1 \otimes B_{+}+B_{+} \otimes 1 \equiv \Delta_{0}\left(B_{+}\right) .
\end{aligned}
$$

On the other hand, we find that

$$
\left[-z A_{+} \otimes N,\left[\ldots\left[-z A_{+} \otimes N, \Delta_{0}\left(B_{+}\right)\right]\right]^{n)} \ldots\right]=\left(-2 z A_{+}\right)^{n} \otimes B_{+} \quad n \geq 1
$$

and the proof for $B_{+}$follows

$$
\begin{array}{r}
e^{-z A_{+} \otimes N} \Delta_{0}\left(B_{+}\right) e^{z A_{+} \otimes N}=\Delta_{0}\left(B_{+}\right)+\sum_{n=1}^{\infty} \frac{\left(-2 z A_{+}\right)^{n}}{n !} \otimes B_{+} \\
=1 \otimes B_{+}+B_{+} \otimes 1+\left(e^{-2 z A_{+}}-1\right) \otimes B_{+}=\sigma \circ \Delta\left(B_{+}\right) .
\end{array}
$$

We consider now $X \equiv B_{-}$and $f \equiv z N \otimes A_{+}$; thus we have

$$
\begin{aligned}
& {\left[z N \otimes A_{+}, \Delta\left(B_{-}\right)\right]=z N \otimes\left\{e^{z A_{+}}\left(z M N-A_{-}\right)-A_{-}\right\}-z^{2} N^{2} \otimes e^{z A_{+}} M} \\
& \quad-2 z B_{-} \otimes e^{2 z A_{+}} A_{+}-z^{2} A_{-} N \otimes e^{2 z A_{+}} A_{+} \\
& \quad+z A_{-} N \otimes\left(e^{2 z A_{+}}-e^{z A_{+}}\right)+z^{2} A_{-} \otimes e^{z A_{+}} A_{+} N \\
& {\left[z N \otimes A_{+},\left[z N \otimes A_{+}, \Delta\left(B_{-}\right)\right]\right]=2 z^{2} N^{2} \otimes e^{z A_{+}} M+4 z^{2} B_{-} \otimes e^{2 z A_{+}} A_{+}^{2}} \\
& \quad+3 z^{3} A_{-} N \otimes e^{2 z A_{+}} A_{+}^{2}-2 z^{2} A_{-} N \otimes\left(e^{2 z A_{+}}-e^{z A_{+}}\right) A_{+}-z^{3} A_{-} \otimes e^{z A_{+}} A_{+}^{2} N .
\end{aligned}
$$

In general, a recurrence method gives (for $n \geq 3$ ):

$$
\begin{aligned}
& {\left[z N \otimes A_{+},\left[\ldots\left[z N \otimes A_{+}, \Delta\left(B_{-}\right)\right]\right]^{n)} \ldots\right]=B_{-} \otimes e^{2 z A_{+}}\left(-2 z A_{+}\right)^{n}} \\
& +z\left(2^{n}-1\right) A_{-} N \otimes e^{2 z A_{+}}\left(-z A_{+}\right)^{n}+z n A_{-} N \otimes\left(e^{2 z A_{+}}-e^{z A_{+}}\right)\left(-z A_{+}\right)^{n-1} \\
& -z A_{-} \otimes e^{z A_{+}}\left(-z A_{+}\right)^{n} N .
\end{aligned}
$$

From (A.7 A.9), the following result is obtained:

$$
\begin{aligned}
& g=e^{z N \otimes A_{+}} \Delta\left(B_{-}\right) e^{-z N \otimes A_{+}}=\Delta\left(B_{-}\right)+z N \otimes\left\{e^{z A_{+}}\left(z M N-A_{-}\right)-A_{-}\right\} \\
& +\sum_{n=1}^{\infty} B_{-} \otimes e^{2 z A_{+}} \frac{\left(-2 z A_{+}\right)^{n}}{n !}+z \sum_{n=1}^{\infty} A_{-} N \otimes e^{2 z A_{+}}\left(\frac{\left(-2 z A_{+}\right)^{n}}{n !}-\frac{\left(-z A_{+}\right)^{n}}{n !}\right)
\end{aligned}
$$




$$
\begin{aligned}
& +z \sum_{n=0}^{\infty} A_{-} N \otimes\left(e^{2 z A_{+}}-e^{z A_{+}}\right) \frac{\left(-z A_{+}\right)^{n}}{n !}-z \sum_{n=1}^{\infty} A_{-} \otimes e^{z A_{+}} \frac{\left(-z A_{+}\right)^{n}}{n !} N \\
& =1 \otimes B_{-}+B_{-} \otimes e^{2 z A_{+}}-z A_{-} \otimes e^{z A_{+}} N-z N \otimes A_{-} \\
& +B_{-} \otimes e^{2 z A_{+}}\left(e^{-2 z A_{+}}-1\right)+z A_{-} N \otimes e^{2 z A_{+}}\left(e^{-2 z A_{+}}-e^{-z A_{+}}\right) \\
& +z A_{-} N \otimes\left(e^{2 z A_{+}}-e^{z A_{+}}\right) e^{-z A_{+}}-z A_{-} \otimes e^{z A_{+}}\left(e^{-z A_{+}}-1\right) N \\
& =1 \otimes B_{-}+B_{-} \otimes 1-z N \otimes A_{-}-z A_{-} \otimes N .
\end{aligned}
$$

Now, we have to compute (A.2) with $f \equiv-z A_{+} \otimes N$ and $g$ instead of $\Delta(X)$. In particular,

$$
\begin{aligned}
& {\left[-z A_{+} \otimes N, g\right]=z\left\{e^{z A_{+}}\left(A_{-}-z M N\right)+A_{-}\right\} \otimes N-z^{2} M e^{z A_{+}} \otimes N^{2}} \\
& \quad+2 z A_{+} \otimes B_{-}+z^{2} A_{+} \otimes A_{-} N-z\left(e^{z A_{+}}-1\right) \otimes A_{-} N-z^{2} A_{+} N \otimes A_{-} \\
& {\left[-z A_{+} \otimes N,\left[-z A_{+} \otimes N, g\right]\right]=2 z^{2} M e^{z A_{+}} \otimes N^{2}+4 z^{2} A_{+}^{2} \otimes B_{-}} \\
& \quad+3 z^{3} A_{+}^{2} \otimes A_{-} N-2 z^{2} A_{+}\left(e^{z A_{+}}-1\right) \otimes A_{-} N-z^{3} A_{+}^{2} N \otimes A_{-}
\end{aligned}
$$

and for $n \geq 3$ :

$$
\begin{aligned}
& {\left[-z A_{+} \otimes N,\left[\ldots\left[-z A_{+} \otimes N, g\right]\right]^{n)} \ldots\right]=z\left(2^{n}-1\right)\left(z A_{+}\right)^{n} \otimes A_{-} N} \\
& +\left(2 z A_{+}\right)^{n} \otimes B_{-}-z n\left(z A_{+}\right)^{n-1}\left(e^{z A_{+}}-1\right) \otimes A_{-} N-z\left(z A_{+}\right)^{n} N \otimes A_{-} .
\end{aligned}
$$

In this way, we complete the proof:

$$
\begin{aligned}
& e^{-z A_{+} \otimes N} g e^{z A_{+} \otimes N}=g+z\left\{e^{z A_{+}}\left(A_{-}-z M N\right)+A_{-}\right\} \otimes N \\
& +z \sum_{n=1}^{\infty}\left(\frac{\left(2 z A_{+}\right)^{n}}{n !}-\frac{\left(z A_{+}\right)^{n}}{n !}\right) \otimes A_{-} N+\sum_{n=1}^{\infty} \frac{\left(2 z A_{+}\right)^{n}}{n !} \otimes B_{-} \\
& -z \sum_{n=0}^{\infty} \frac{\left(z A_{+}\right)^{n}}{n !}\left(e^{z A_{+}}-1\right) \otimes A_{-} N-z \sum_{n=1}^{\infty} \frac{\left(z A_{+}\right)^{n}}{n !} N \otimes A_{-} \\
& =1 \otimes B_{-}+B_{-} \otimes 1-z N \otimes A_{-}+z e^{z A_{+}}\left(A_{-}-z M N\right) \otimes N \\
& +z\left(e^{2 z A_{+}}-e^{z A_{+}}\right) \otimes A_{-} N+\left(e^{2 z A_{+}}-1\right) \otimes B_{-} \\
& -z e^{z A_{+}}\left(e^{z A_{+}}-1\right) \otimes A_{-} N-z\left(e^{z A_{+}}-1\right) N \otimes A_{-}=\sigma \circ \Delta\left(B_{-}\right) .
\end{aligned}
$$

Finally, notice that $\mathcal{R}^{-1}=\sigma \circ \mathcal{R}$, that is, $\mathcal{R}$ is a triangular $R$-matrix.

\section{References}

[1] Zhang W-M, Feng D H and Gilmore R 1990 Rev. Mod. Phys. 62867

[2] Brif C 1996 Ann. Phys. 251180

[3] Ballesteros A and Herranz F J 1996 J. Phys. A: Math. Gen. 294307

[4] Floreanini R and Vinet L 1994 Lett. Math. Phys. 3237

[5] Dobrev V K, Doebner H-D and Mrugalla C 1996 J. Phys. A: Math. Gen. 29 5909 
[6] Chari V and Pressley A 1995 A guide to Quantum Groups, Cambridge University Press

[7] Ballesteros A, Herranz F J, del Olmo M A, Pereña C M and Santander M 1995 J. Phys. A: Math. Gen. 287113

[8] Ballesteros A and Herranz F J 1996 J. Phys. A: Math. Gen. 29 L311

[9] Ballesteros A, Herranz F J and Negro J Boson representations, non-standard quantum algebras and contractions, J. Phys. A: Math. Gen. to appear

[10] Celeghini E, Rasetti M and Vitiello G 1995 J. Phys. A: Math. Gen. 28 L239

[11] Dobrev V K Representations of the Jordanian quantum algebra $U_{h}(s l(2))$, Proc. 10th Int. Conf. 'Problems of Quantum Field Theory', eds. D. Shirkov, D. Kazakov and A. Vladimirov, JINR E2-96-369, (Dubna, 1996) pp. 104; preprint ICTP $\mathrm{IC} / 96 / 14$

[12] Abdesselam B, Chakrabarti A and Chakrabarti R 1996 Mod. Phys. Lett. 11A 2883

[13] Abdesselam B, Chakrabarti A and Chakrabarti R 1997 Int. J. Mod. Phys. 12A 2301

[14] Aizawa N Irreducible decomposition for tensor product representations of Jordanian quantum algebras, J. Phys. A: Math. Gen. to appear

[15] Van der Jeugt J Representations and Clebsch-Gordan coefficients for the Jordanian quantum algebra $U_{h}(\operatorname{sl}(2))$ q-alg/9703011

[16] Fock V A 1928 Z. Phys. 49 339; Bargmann V 1961 Comm. Pure Appl. Math 14187

[17] Hagen C R 1972 Phys. Rev. D 5377

[18] Niederer U 1972 Helv. Phys. Acta 45802

[19] Negro J 1997, private communication 